Nephrologe 2014 • 9:9-10

DOI 10.1007/s11560-013-0767-7

Online publiziert: 27. Januar 2014

(c) Springer-Verlag Berlin Heidelberg 2014

\author{
J. Floege ${ }^{1} \cdot$ J. Hoyer ${ }^{2}$ \\ ${ }^{1}$ Medizinische Klinik II, Rheinisch-Westfälische Technische Hochschule Aachen \\ 2 Klinik für Innere Medizin, Schwerpunkt Nephrologie, Universitätsklinikum \\ Gießen und Marburg GmbH, Standort Marburg
}

\title{
Geriatrische Nephrologie
}

Der demographische Wandel hat erhebliche Auswirkungen auf das Gesundheitssystem und fordert eine adäquate Anpassung der medizinischen Versorgung an die spezifischen Bedürfnisse älterer Patienten. Die Alterung der Gesellschaft wirkt sich verstärkt auf Fachgebiete mit einem hohem Anteil an chronisch kranken Patienten aus. So sind besonders auch wir Nephrologen aufgefordert, unser diagnostisches und therapeutisches Verhalten für diese Patientengruppe anzupassen und altersadaptierte Behandlungsstrategien zu entwickeln. Leider konnten in vielen klinischen Studien geriatrische Patienten nicht eingeschlossen werden, sodass die therapeutischen Empfehlungen nur mit einer begrenzten Evidenz belegt sind.

In diesem Themenschwerpunkt werden zentrale klinische Aspekte aus der geriatrischen Nephrologie dargestellt.

Die Nieren unterliegen nicht nur einem physiologischen Alterungsprozess mit progredienter Minderung des funktionellen Nierengewebes. Mit zunehmendem Alter weisen sie auch eine erheblich verminderte Regenerationsfähigkeit nach einer Gewebeschädigung auf. Dies macht die Nieren auf spezielle Weise vulnerabel für die Schädigungen durch die bei älteren Patienten häufigen Komorbiditäten Diabetes mellitus, Hypertonus und Atherosklerose. Die Kolleginnen Banas, Amann und Schaeffner beschreiben die Mechanismen der physiologischen Alterungsprozesse der Nieren und zeigen, welche Perspektiven die Kenntnis von renaler zellulärer Seneszenz und deren morphologischer Manifestation für die Entwicklung präventiver Maßnahmen, die Identifizierung spezifischer Medikamentenwirkungen und die Entwicklung altersadaptierter Therapieschemata haben kann.
Die alltägliche Behandlungspraxis älterer Nierenpatienten unterliegt vielfältigen Besonderheiten. Wie Breninkmeijer und Busch zeigen, sind hierbei altersspezifische Änderungen in der Inzidenz und im klinischen Erscheinungsbild renaler Erkrankungen zu beachten. Das vermehrte Auftreten von kardiovaskulären Komplikationen bei Nierenpatienten macht eine Anpassung von präventiven Maßnahmen, z. B. Lipidsenkertherapie oder Antikoagulanzien, notwendig und stellt besondere Anforderungen an die Indikation für interventionelle Therapien. Gleichfalls bedarf die verstärkte Neigung zu Harnwegsinfektionen alters- und geschlechtsspezifischer Behandlungsweisen. Die bei älteren Patienten häufig notwendige Polypharmazie hat erhebliche Auswirkungen auf die Pharmakodynamik und -kinetik, sodass es spezifischer Anpassungen in der medikamentösen Therapie bedarf.

\section{》) Die Nieren sind besonders vulnerabel für Schädigungen durch die bei älteren Patienten häufigen Komorbiditäten}

Die renale Osteopathie ist für ältere Nierenpatienten wegen der schmerzhaften Symptomatik und des hohen Frakturrisikos von großer Bedeutung für die Lebensqualität. Auf dem Gebiet der renalen Knochenerkrankungen konnte in den letzten Jahren durch eine Reihe von klinischen wie experimentellen Studien die Regulation des Knochenstoffwechsels durch Faktoren wie FGF23, BMP2 und PTH detailliert charakterisiert werden. Daraus konnte eine Reihe neuer Pharmaka zur Behandlung der renalen Osteopathie entwickelt werden, welche Eingang in die klinische Praxis fanden. Auf der Basis ak- tueller Daten fassen Ketteler und Schlieper die aktuellen Empfehlungen (bzw. die Wissenslücken ...) für die Behandlung für Störungen des Kalzium-Phosphat-Stoffwechsels zusammen.

Im Vergleich zur Gesamtbevölkerung sind Dialysepatienten in hohem Maß von einer Minderung der kognitiven Leistungsfähigkeit bis hin zur manifesten demenziellen Erkrankung betroffen. Diese wird verstärkt durch eine übermäßig hohe Inzidenz an depressiven Erkrankungen bei Dialysepatienten. Beide Krankheiten sind jedoch bei Dialysepatienten unterdiagnostiziert: Eine Demenz wird in weniger als $1 \%$ der über 60-jährigen Dialysepatienten diagnostiziert, im Gegensatz zu etwa $6 \%$ in der Gesamtbevölkerung. Bereits einfache Screening-Tests können eine eingeschränkte von einer uneingeschränkten Kognition unterscheiden. Spezifische Testungen können zudem Hinweise auf die präferenzielle Einschränkung von Gedächtnisleistungen oder exekutiven Hirnleistungen geben.

In ihrem Beitrag heben Karakizlis und Mühlfeld hervor, dass die diagnostische Kenntnis von Demenz oder Depression direkte therapeutische Implikationen z. B. auf die individualisierte Pharmakotherapie hat. Die Einschätzung des kognitiven Vermögens eines Patienten hat zudem Bedeutung für das gesamte nephrologische Team, da missverständliche Situationen oder Compliance-Probleme besser realisiert und ggf. vermieden werden können. Anforderungen an den Patienten können angepasst und komplexere Aufgaben in der Diätetik oder der Medikation eher an Angehörige oder Pflegende weitergegeben werden.

In die Behandlung älterer oder hochbetagter Patienten mit Niereninsuffizienz bzw. älterer Dialysepatienten sind über 
die internistisch-nephrologischen Inhalte hinaus besondere medizinische und psychologische Aspekte einzubeziehen. Bei jedem Patienten ist eine abgewogene Einschätzung seiner körperlichen Möglichkeiten notwendig - besonders da eine Vielzahl älterer Patienten einen außerordentlich guten körperlichen Zustand aufweist. Letztendlich müssen dabei die Grenzen sinnvollen medizinischen Handelns reflektiert werden. Eine Dialysetherapie kann beim älteren Patienten nicht isoliert als Nierenersatzverfahren betrachtet werden. Vielmehr sollten die Therapieempfehlungen und ggf. die Empfehlung für ein Dialyseverfahren mit Blick auf den Gesamtkontext des Patienten erfolgen. Im Spannungsfeld von Machbarkeit und Empfehlung fassen Kleophas, Kuhlmann und Pommer die ethischen Aspekte der Behandlung von älteren Nierenpatienten zusammen. Mit dem Leitsatz „Add more life to years, not more years to life" geben sie eine Basis für Verhaltensweisen in der geriatrischen Nephrologie.

Die Beiträge des Themenschwerpunkts sollen Ihnen einen Einblick in die spezielle Problematik der geriatrischen Nephrologie geben. Sie möchten zu einer inhaltlichen Positionierung der Nephrologie in der Versorgung älterer oder betagter Patienten beitragen, welche sowohl für die tägliche Arbeit des medizinischen Personals als auch für das Befinden der $\mathrm{Pa}$ tienten von großer Bedeutung sein kann.
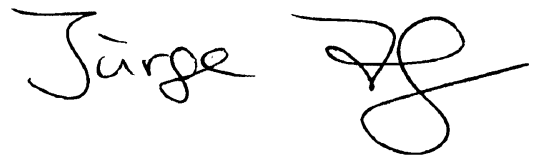

Jürgen Floege

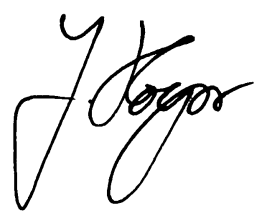

Joachim Hoyer

\section{Korrespondenzadressen}

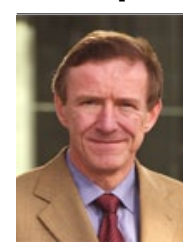

Prof. Dr. J. Floege

Medizinische Klinik II, Rheinisch-Westfälische Technische Hochschule Aachen Pauwelsstr. 30, 52057 Aachen juergen.floege@ rwth-aachen.de

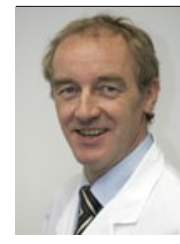

\section{Prof. Dr. J. Hoyer}

Klinik für Innere Medizin, Schwerpunkt Nephrologie, Universitätsklinikum Gießen und Marburg GmbH, Standort Marburg Baldingerstr., 35043 Marburg hoyer@med.uni-marburg.de

Interessenkonflikt. J. Floege und J. Hoyer geben an dass kein Interessenkonflikt besteht.

\section{Arteriosklerose-Artikel online frei zugänglich}

Hierzulande ist Arteriosklerose eine der häufigsten Gefäßerkrankungen; 18\% der Frauen und 28\% der Männer in der Altersgruppe über 65 Jahre sind betroffen. Im Zuge der ACTION (Arteriosklerose -

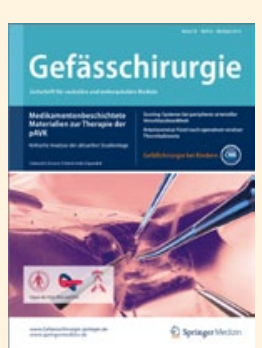
Circulation und Training InformatiOns Netzwerk)Kampagne der Deutschen Gesellschaft für Gefäßchirurgie und Gefäßmedizin (DGG) stehen zwei

Übersichtsarbeiten zu Arteriosklerose für einen breiten Leserkreis online frei zugänglich bereit.

Im ersten Beitrag in Ausgabe 6/13 von Gefässchirurgie beschreiben die Autoren Risikofaktoren, Epidemiologie und die Ursachen von Arteriosklerose, im zweiten Beitrag in Ausgabe 7/13 von Gefässchirurgie erläutern sie die Manifestation der Arteriosklerose und die Bedeutung von Präventionsmaßnahmen.

\section{Ursachen und Risikofaktoren der Arteriosklerose}

dx.doi.org/10.1007/s00772-013-1233-6

Manifestation und Prävention der Arteriosklerose

dx.doi.org/10.1007/s00772-013-1235-4

Mit der ACTION -Kampagne trägt die Deutsche Gesellschaft für Gefäßchirurgie und Gefäßmedizin (DGG) zur Aufklärung, Information und Prävention über Arteriosklerose bei.

Suchen Sie noch mehr zum Thema? Mit e.Med, dem Online-Paket von Springer Medizin, können Sie schnell und komfortabel in über 500 medizinischen Fachzeitschriften recherchieren.

Weitere Infos unter: springermedizin.de/eMed. 